\title{
PROMOÇÃO DA SAÚDE E TUBERCULOSE
}

\author{
Mônica Mussolini Larroque ${ }^{1}$ \\ Branca Maria de Oliveira Santos ${ }^{2}$
}

LARroque, M. M.; SANTOS, B. M. de O. Promoção da saúde e tuberculose. Arq. Cienc. Saúde UNIPAR, Umuarama, v. 19, n. 3, p, 221-228, set./dez. 2015.

RESUMO: A tuberculose (TB) é uma doença antiga que acompanha a humanidade há milênios. Apesar dos vários esforços para seu controle e eliminação os indicadores da doença são altos, especialmente nos países com precárias condições de vida. Objetivando realizar uma revisão sistemática da literatura para identificar pesquisas que associem algum indicador epidemiológico da tuberculose com as condições de vida da população, foram realizadas buscas eletrônicas em publicações nos bancos de dados Scientific Electronic Library Online (SCIELO) e na Biblioteca Regional de Medicina (BIREME). Das publicações selecionadas 9 (52,9\%) foram estudos ecológicos com representação espacial, $6(35,3 \%)$ utilizaram como método a pesquisa qualitativa com utilização de entrevistas e $2(11,8 \%)$ foram estudos epidemiológicos descritivos. Os aspectos socioeconômicos como índice de desenvolvimento humano, renda, emprego/ocupação, condições de moradia, quantidade de pessoas por residência e o acesso aos serviços de saúde foram os mais frequentemente utilizados. Após décadas de tentativas de controle e eliminação da doença, o país vive uma fase de estagnação da qual dependem estratégias e políticas mais amplas voltadas à promoção da saúde, para superar não só os altos indicadores epidemiológicos da tuberculose, como de várias doenças. PALAVRAS-CHAVE: Tuberculose; Promoção da saúde; Epidemiologia.

\section{HEALTH PROMOTION AND TUBERCULOSIS}

ABSTRACT: Tuberculosis (TB) is an ancient disease that accompanies humankind for millennia. Despite various efforts for its control and elimination, the disease indicators are high, especially in countries with poor living conditions. Aiming at performing a systematic review of the literature to identify studies involving tuberculosis epidemiological indicator related to the population living conditions, electronic searches were conducted in publications in the databases of Scientific Electronic Library Online (SciELO) and the Regional Library of Medicine (BIREME). From the publications selected, nine (52.9\%) were ecological studies with spatial representation, six (35.3\%) used a qualitative research as their method with the use of interviews, and two (11.8\%) were descriptive epidemiological studies. The socio-economic aspects such as the human development index, income, employment/occupation, housing conditions, number of people per household and access to health services were the most frequent aspects used. After decades of attempts to control and eliminate the disease, the country is experiencing a period of stagnation that depends on broader strategies and policies aimed at promoting health, in order to overcome not only the high epidemiological indicators of tuberculosis, but also various other diseases.

KEYWORDS: Tuberculosis; Health promotion; Epidemiology.

\section{Introdução}

A promoção da saúde objetiva introduzir ações e políticas voltadas ao bem-estar individual e coletivo por meio de uma intensa organização social, de modo que todos tenham oportunidade de se desenvolver ao longo da vida de maneira saudável, mantendo e melhorando suas condições de saúde (BRASIL, 2006).

Segundo Buss (2000), a promoção da saúde está ligada principalmente à evolução e mudanças dos comportamentos das pessoas. Para ele os indivíduos passam a ter maior conhecimento e domínio sobre os seus determinantes sociais e, consequentemente, melhoram sua situação de saúde. Isso significa que os aspectos relacionados à alimentação/ nutrição, emprego/renda, habitação, educação e ambiente físico e social passam a ser conhecidos pelos indivíduos e melhor controlados por esses a fim de aumentar a qualidade de vida e de saúde das coletividades.

Para o SUS a promoção da saúde funciona como uma estratégia de produção de ações de modo articulado às demais políticas já existentes com a finalidade de responder as demandas sociais em saúde da população (BRASIL, 2007).
Uma das doenças que desencadeiam graves problemas sociais e para a saúde pública é a tuberculose (TB), pois acomete principalmente pessoas em idade produtiva e desfavorecidas social e economicamente. Em geral, os indivíduos possuem baixa renda familiar, educação precária, habitação ruim ou inexistente, famílias numerosas, desnutrição, alcoolismo, vivem em agrupamentos comunitários, possuem outras doenças infecciosas associadas e dificuldade de acesso aos serviços de saúde, evidenciando que as ações de controle não se resumem apenas ao setor da saúde.

Estar exposto a um indivíduo doente é um requisito para ser infectado, e uma vez exposto vários fatores determinarão o risco de se contrair a infecção. Da mesma forma, uma pessoa infectada depende de vários outros fatores de risco para desenvolver a doença. O conjunto desses fatores caracteriza, entre outros, os determinantes sociais de saúde que para a tuberculose são fortemente relacionados às condições de vida e vulnerabilidade social (KRITSKI; CONDE; SOUZA, 2005).

A tuberculose tem sido identificada em várias raças e povos nos mais diversos continentes. Mundialmente os casos estão relacionados com a pobreza, má distribuição de renda e a urbanização acelerada. A epidemia do vírus da imu-

DOI: https://doi.org/10.25110/arqsaude.v19i3.2015.5552

${ }^{1}$ Pós-Graduanda de doutorado do Programa de Promoção da saúde da Universidade de Franca - UNIFRAN. Docente da Graduação em Medicina - Universidade Federal de Mato Grosso do Sul - Três Lagoas - Mato Grosso do Sul. Endereço para correspondência: Mônica Mussolini Larroque. Universidade Federal de Mato Grosso do Sul. Campus II Av. Ranulpho Marques Leal, 3.484 - Caixa Postal 210, CEP 79620-080 Fone: (67) 3509-3700 Fax: (67) 3509-3700 Três Lagoas, MS. email: monicamussolini@hotmail.com

${ }^{2}$ Docente da Pós Graduação em Promoção da Saúde -Universidade de Franca - Unifran - Franca - São Paulo. Endereço para correspondência: Rua Imbuia, n. 25. CEP 14040-200. Fone: (16) 36307877. Ribeirão Preto, SP. E-mail: brancamosantos@gmail.com 
nodeficiência humana (HIV) e da síndrome da imunodeficiência adquirida (AIDS) e o aparecimento do Mycobacterium tuberculosis multirresistente, têm alertado o mundo para a necessidade de medidas mais eficazes no controle da doença (VENDRAMINI et. al., 2005; WORLD HEALTH ORGANIZATION, 2006).

O Plano Global de Controle da Tuberculose previsto para o período de 2006-2015, organizado pela Organização Mundial da Saúde (OMS), tem como objetivo primordial reduzir a carga mundial da TB (incidência, prevalência e mortalidade). A despeito da conscientização das autoridades de saúde e dos programas de prevenção e controle no Brasil, a doença continua como um importante problema de saúde pública e estima-se uma prevalência de 50 milhões de indivíduos infectados pelo Mycobacterium tuberculosis, com surgimento anual de 130.000 casos novos e 6.000 óbitos (BRASIL, 2010; SANTOS, 2007; WORLD HEALTH ORGANIZATION, 2006).

Os principais determinantes da magnitude e persistência da tuberculose no país são: as desigualdades sociais; a urbanização acelerada e desordenada; a existência de processos migratórios; bem como a debilidade do sistema de saúde e suas deficiências de gestão. Assim, são necessárias ações constantes fundamentadas pela promoção da saúde e intersetorialidade das ações, para que se alcance o sucesso esperado na prevenção e controle da doença (BRASIL, 2002; MOREIRA et al., 2008).

Compreendendo a importância da tuberculose no Brasil e no mundo e, diante das recentes pesquisas a respeito dela em vários estados brasileiros, este é um estudo importante, pois apresenta as condições de vida e sua relação direta com a promoção da saúde e a tuberculose.

Este artigo objetiva realizar uma revisão sistemática da literatura, identificando pesquisas que associem algum indicador epidemiológico da tuberculose com as condições de vida, e aponta que após décadas de tentativas de controle e eliminação da deste agravo no país, vivemos uma fase de estagnação da qual dependem estratégias e políticas mais amplas voltadas à promoção da saúde, para superar não só os altos indicadores epidemiológicos da tuberculose, como de várias outras doenças.

\section{Material e Método}

Trata-se de uma revisão sistemática da literatura. As publicações identificadas neste estudo são de pesquisas realizadas no Brasil que apresentam associação entre indicadores da tuberculose e condições de vida, tais como: renda/emprego, escolaridade, condições de moradia, acesso aos serviços de saúde, qualidade de vida e vulnerabilidades.

\section{Estratégia de pesquisa}

As publicações foram selecionadas em dois bancos de dados: o Scientific Electronic Library Online (SCIELO) e no Centro Latino-Americano e do Caribe de Informação em Ciências da Saúde, também conhecido como Biblioteca Regional de Medicina (BIREME). Utilizaram-se as seguintes palavras chaves e suas combinações: "tuberculose" (tuberculosis), "promoção da saúde" (health promotion), "economia" (economic) "demografia" (demography), cultura/aspectos culturais (culture), "social/aspectos sociais" (social) e "educação" (education). Todos os termos foram consultados e são registrados nos Descritores em Ciências da Saúde (DeCS).

\section{Critérios de inclusão}

Foram incluídas as publicações disponíveis na íntegra, ou seja, textos completos e disponíveis gratuitamente nas bases de dados selecionadas, entre o período de janeiro de 2010 a dezembro de 2014 e nos idiomas português e inglês.

\section{Critérios de exclusão}

As pesquisas excluídas foram as que não relacionaram condições de vida aos indicadores da tuberculose ou que apresentaram apenas algum indicador da TB. Foram excluídos também estudos que utilizaram na metodologia apenas a modalidade de revisão da literatura, aquelas realizadas com indicadores e condições de vida de outros países, em outro idioma ou que possuam apenas o resumo e não o texto completo disponível.

\section{Resultados e Discussão}

A busca entre as associações dos descritores selecionados nas bases de dados, em conformidade com os critérios de inclusão, encontrou um total de 635 publicações científicas das quais, 204 na base de dados Scielo e 431 na base de dados BIREME.

A figura 1 apresenta o fluxograma utilizado para a seleção das publicações por etapas.

Figura 1: Representação gráfica das etapas de seleção das publicações e seus respectivos resultados.

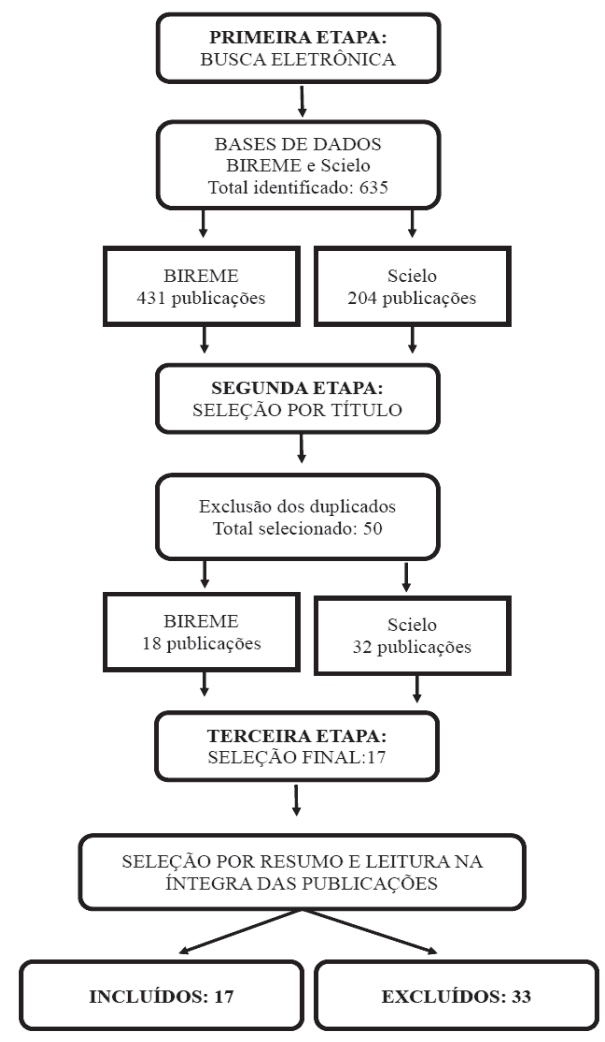


Após a primeira etapa de busca eletrônica foi realizada uma seleção das publicações de acordo com o título. $\mathrm{Na}$ segunda etapa foram excluídos os estudos duplicados, aqueles que não apresentavam o trabalho na íntegra e os realizados em outros países. Após esta seleção foram encontradas 50 publicações.

Para a fase final foram lidos todos os resumos e os textos na íntegra. Nesta fase foram selecionadas as $17 \mathrm{pu}-$ blicações sendo 15 em português e 2 em inglês. Todas as publicações que compõem este trabalho possuem alguma relação entre promoção da saúde e/ou qualidade de vida e a tuberculose.

As publicações selecionadas foram organizadas quanto ao autor, ano de publicação, local, métodos utilizados no estudo, indicador epidemiológico citado, associação entre os indicadores e aspectos da promoção da saúde, resultados e discussão da pesquisa e conclusão (Quadro 1).

Das publicações selecionadas $9(52,9 \%)$ foram estudos ecológicos com representação espacial, 6 (35,3\%) utilizaram como método a pesquisa qualitativa com utilização de entrevistas e $2(11,8 \%)$ foram estudos epidemiológicos descritivos.

Os aspectos socioeconômicos como índice de de- senvolvimento humano, renda, emprego/ocupação, condições de moradia, quantidade de pessoas por residência, receber auxílio ou participar de política pública social (como Bolsa Família, por exemplo) e o acesso aos serviços de saúde foram frequentemente utilizados para relacionar a doença às condições de vida das populações de estudo, evidenciando a preocupação dos autores em identificar questões relacionadas à promoção da saúde.

\section{Relação entre indicadores epidemiológicos da tuberculo- se e aspectos socioeconômicos}

As publicações trabalham com dados de condições de vida e revelam que pessoas que possuem carências nutricionais e vivem em condições precárias de moradia, ambientes e cômodos superlotados e pouco ventilados, possuem maiores riscos de adoecer (PIVA et al., 2013; ROZA; CACCIA-BAVA; MARTINEZ, 2012).

Segundo Hino et al. (2011a), Neves et al. (2012) e Vendramini et al. (2010) a TB ultrapassa as barreiras biológicas, pois, é uma doença social ligada à pobreza, à privação social e à marginalidade fruto de um espaço interurbano desigual, fragmentado e fortemente excludente.

Tabela 1: Distribuição das publicações selecionadas segundo autor, ano, local, método utilizado na pesquisa, indicador epidemiologico, aspectos da promoção da saúde, resultados/discussão e conclusão.

\begin{tabular}{|c|c|c|c|c|c|}
\hline Autor (Ano) Local & $\begin{array}{l}\text { Métodos da } \\
\text { pesquisa }\end{array}$ & $\begin{array}{c}\text { Indicador } \\
\text { Epidemiológico }\end{array}$ & $\begin{array}{c}\text { Associação } \\
\text { aos aspectos } \\
\text { de promoção } \\
\text { da saúde* }\end{array}$ & $\begin{array}{l}\text { Resultados e } \\
\text { discussão }\end{array}$ & Conclusão \\
\hline $\begin{array}{l}\text { WELCH; COIM- } \\
\text { BRA, (2011) } \\
\text { Rio de Janeiro - RJ }\end{array}$ & $\begin{array}{l}\text { Qualitativa com } \\
\text { realização de entre- } \\
\text { vistas. }\end{array}$ & $\begin{array}{l}\text { Incidência anual } \\
\text { média: } 1.200 \text { por } \\
100.000 \text {. } \\
* \text { nacional é de } 41,3 \\
\text { por } 100.000\end{array}$ & $\begin{array}{l}\text { A.C. } \\
\text { A.S. }\end{array}$ & $\begin{array}{l}\text { A TB possui caráter } \\
\text { fortemente cultural. } \\
\text { Acreditam em dois } \\
\text { tipos da doença: a } \\
\text { TB microbiológica e } \\
\text { a TB feitiçaria. }\end{array}$ & $\begin{array}{l}\text { Para o autor a intercultu- } \\
\text { ralidade não é impeditivo } \\
\text { das medidas de controle } \\
\text { preconizadas e sim a falta } \\
\text { de estrutura da rede de saú- } \\
\text { de local. }\end{array}$ \\
\hline $\begin{array}{l}\text { LEMOS et al. } \\
(2012) \\
\text { Fortaleza - CE }\end{array}$ & $\begin{array}{l}\text { Qualitativa com } \\
\text { aplicação da escala } \\
\text { de qualidade de vida } \\
\text { denominada HAT- } \\
\text {-Qol. }\end{array}$ & $\begin{array}{l}\text { Coinfecção tubercu- } \\
\text { lose/ HIV. }\end{array}$ & $\begin{array}{l}\text { Q.V. } \\
\text { A.S. } \\
\text { V. }\end{array}$ & $\begin{array}{l}\text { A maioria dos entre- } \\
\text { vistados relata pre- } \\
\text { juízo na qualidade } \\
\text { de vida, afetando o } \\
\text { bem-estar físico, so- } \\
\text { cial e psicológico. }\end{array}$ & $\begin{array}{l}\text { Principais aspectos preju- } \\
\text { diciais à qualidade de vida } \\
\text { são econômicos, preocupa- } \\
\text { ção com a saúde e sigilo da } \\
\text { doença. }\end{array}$ \\
\hline $\begin{array}{l}\text { HINO et al. (2011a) } \\
\text { São Paulo - SP }\end{array}$ & $\begin{array}{l}\text { Qualitativa com } \\
\text { realização de entre- } \\
\text { vistas. }\end{array}$ & Morbidade. & $\begin{array}{l}\text { Q.V. } \\
\text { A.S. } \\
\text { A.E. } \\
\text { V. }\end{array}$ & $\begin{array}{l}\text { Doença trouxe } \\
\text { medo, angústia, } \\
\text { isolamento social, } \\
\text { sintomas físicos im- } \\
\text { portantes, estigma. }\end{array}$ & $\begin{array}{l}\text { Atenção ao vínculo, à } \\
\text { valorização das queixas e } \\
\text { atenção as vulnerabilidades } \\
\text { para garantir atendimento } \\
\text { humanizado. }\end{array}$ \\
\hline $\begin{array}{l}\text { ROZA; CACCIA- } \\
\text {-BAVA; MARTI- } \\
\text { NEZ, (2012). } \\
\text { Ribeirão Preto-SP }\end{array}$ & Estudo ecológico. & $\begin{array}{l}\text { Número de casos } \\
\text { com vulnerabilidade } \\
\text { social, renda e edu- } \\
\text { cação em Ribeirão } \\
\text { Preto SP, }\end{array}$ & $\begin{array}{l}\text { V. } \\
\text { Q.V. } \\
\text { A.E. } \\
\text { A.S. } \\
\text { E. }\end{array}$ & $\begin{array}{l}\text { Áreas de risco } \\
\text { elevado e de vul- } \\
\text { nerabilidade social. } \\
\text { Encontrou áreas } \\
\text { com baixa vulnera- } \\
\text { bilidade e alta es- } \\
\text { colaridade e renda, } \\
\text { mas com altas taxas } \\
\text { estimadas de TB. }\end{array}$ & $\begin{array}{l}\text { Segundo o estudo há rela- } \\
\text { ção das taxas com as me- } \\
\text { didas de renda, educação } \\
\text { e vulnerabilidade social, } \\
\text { porém existem outros as- } \\
\text { pectos intrínsecos e fatores } \\
\text { ambientais que merecem } \\
\text { maiores estudos. }\end{array}$ \\
\hline $\begin{array}{l}\text { VENDRAMINI et } \\
\text { al. (2010) } \\
\text { São José do Rio } \\
\text { Preto - SP }\end{array}$ & $\begin{array}{l}\text { Estudo ecológico } \\
\text { utilizando geocodi- } \\
\text { ficação. }\end{array}$ & $\begin{array}{l}\text { Número de casos } \\
\text { novos. Incidência de } \\
\text { TB no município de } \\
34 \text { por } 100.000 .\end{array}$ & $\begin{array}{l}\text { A.S. } \\
\text { A.E. } \\
\text { E. }\end{array}$ & $\begin{array}{l}\text { Controle da doença } \\
\text { depende de interesse } \\
\text { político, da atenção } \\
\text { primária, recursos e } \\
\text { profissionais de saú- } \\
\text { de preparados. }\end{array}$ & $\begin{array}{l}\text { Evidente desigualdade so- } \\
\text { ciogeográfica e áreas com } \\
\text { maiores carências sociais } \\
\text { apresentam maiores inci- } \\
\text { dências. }\end{array}$ \\
\hline
\end{tabular}




\begin{tabular}{lll}
\hline HINO et al. (2011b) & Estudo ecológico & Casos novos notifi- \\
Ribeirão Preto - SP & $\begin{array}{l}\text { com tendência tem- } \\
\text { poral (retrospectivo }\end{array}$ & cados. \\
& e longitudinal)
\end{tabular}

\begin{tabular}{ll}
\hline De SOUZA; da SIL- & Qualitativa com \\
VA; MEIRELLES, & realização de entre- \\
$(2010)$. & vistas.
\end{tabular}

Florianópolis - SC

Morbidade.

A.S. Distribuição não

A.E. uniforme. Os casos

E. de TB concentram

em áreas de maior

pobreza e algumas

de condição intermediária.

$\begin{array}{ll}\text { A.S. } & \text { Viver com Tb é so- } \\ \text { A.C. } & \text { frido, a doença afas- } \\ \text { Q.V. } & \text { ta as pessoas e muda } \\ & \text { a percepção de si. }\end{array}$

Coeficiente de incidência e Índice de

Desenvolvimento

Humano (IDH).

LHO, (2014).

Ribeirão Preto- SP

RODRIGUES-JÚ-

NIOR; RUFFINO-

Estudo ecológico.
YAMAMURA et al. Estudo ecológico. (2014)

São Paulo - SP
Coeficientes de incidência, óbito e abandono.
A.S.

A.E. cidência da doença apresentaram alto IDH. Mudanças sociais e econômicas levam décadas.

A.S. Permitiu verificar

V. que as oportunidades sociais têm colocado pessoas em desvantagem no que tange ao adoecimento por tuberculose.
Regiões com alta in-

A pobreza também pode ser considerada causa da doença, já que esta envolve um sistema complexo que envolve diversos fatores e não apenas o bacilo.

Para apoiar e minimizar os efeitos da doença deve existir ações de promoção da saúde, de educação em saúde e grupos de apoio.

Considerando que os locais de baixa incidência possuem baixo IDH, sugerem melhorias nos sistemas de saúde dessas localidades.

Apesar da organização da atenção à tuberculose, ainda há desafios em utilizá-las para reduzir a iniquidade social, o que seria viabilizado pela interlocução da ESF e dos equipamentos sociais.

Rio de Janeiro - RJ dade.

\section{Estudos que avaliem as} condições de vida são mais interessantes do que aque-

\begin{tabular}{lllll}
\hline GUIMARÃES. et & Estudo ecológico. & $\begin{array}{l}\text { Prevalência, inci- } \\
\text { dência e mortali- }\end{array}$ & $\begin{array}{l}\text { A.S. } \\
\text { A.E. }\end{array}$ & $\begin{array}{l}\text { Relação direta entre } \\
\text { taxas de tuberculose }\end{array}$ \\
$\begin{array}{ll}\text { al. (2012) } \\
\text { Rio de Janeiro - RJ }\end{array}$ & & V. & e taxas de pobreza.
\end{tabular}
les que apresentam apenas indicadores.

\begin{tabular}{lll}
\hline De QUEIROGA et & Estudo ecológico. & Casos novos notifi- \\
al. (2012) & & cados. \\
Campina Grande & & \\
- PB &
\end{tabular}

$\begin{array}{cl}\text { A.S. } & \text { Altas taxas de TB } \\ \text { A.E. } & \text { estão relacionadas } \\ \text { E. } & \text { aos bairros com as }\end{array}$
Estratégias de controle devem ser baseadas em promoção da saúde e qualidade de vida. vida.

\begin{tabular}{lllll}
\hline ACOSTA; BASSA & Estudo ecológico. & Incidência média & A.S. & $\begin{array}{l}\text { Menores taxas na } \\
\text { região central da } \\
\text { NESI, (2014) }\end{array}$ \\
anual. & & A.E. & E. & $\begin{array}{l}\text { cidade e maiores na } \\
\text { periferia. }\end{array}$
\end{tabular}

\begin{tabular}{|c|c|c|c|c|c|}
\hline $\begin{array}{l}\text { ACOSTA; BASSA } \\
\text { NESI, (2014) } \\
\text { Porto Alegre - RS }\end{array}$ & Estudo ecológico. & $\begin{array}{l}\text { Incidência média } \\
\text { anual. }\end{array}$ & $\begin{array}{l}\text { A.S. } \\
\text { A.E. } \\
\text { E. }\end{array}$ & $\begin{array}{l}\text { Menores taxas na } \\
\text { região central da } \\
\text { cidade e maiores na } \\
\text { periferia. }\end{array}$ & $\begin{array}{l}\text { As análises confirmam que } \\
\text { a taxa de incidência da TB } \\
\text { é fortemente correlacionada } \\
\text { com as condições socioeco- } \\
\text { nômicas. Sugere políticas } \\
\text { públicas de redução da } \\
\text { pobreza. }\end{array}$ \\
\hline $\begin{array}{l}\text { De PAULA et al. } \\
\text { (2014) } \\
\text { São Paulo - SP }\end{array}$ & $\begin{array}{l}\text { Qualitativa com } \\
\text { realização de entre- } \\
\text { vistas }\end{array}$ & Morbidade. & $\begin{array}{c}\text { A.C. } \\
\text { E. }\end{array}$ & $\begin{array}{l}\text { Relatam preconcei- } \\
\text { tos e medo de ser } \\
\text { identificado, espera } \\
\text { agravamento dos } \\
\text { sintomas, homens } \\
\text { procuram menos } \\
\text { atendimento, procu- } \\
\text { ram o hospital por } \\
\text { ser mais rápido e a } \\
\text { UBS não é rotina, } \\
\text { assim como a pre- } \\
\text { venção. }\end{array}$ & $\begin{array}{l}\text { Padrões culturais devem ser } \\
\text { levados em conta na cria- } \\
\text { ção de politicas públicas. } \\
\text { Profissionais e gestores de- } \\
\text { vem conhecer o imaginário } \\
\text { popular da qual pretendem } \\
\text { atuar. }\end{array}$ \\
\hline $\begin{array}{l}\text { De OLIVEIRA; } \\
\text { GONÇALVES, } \\
\text { (2013) } \\
\text { Manaus - AM }\end{array}$ & $\begin{array}{l}\text { Estudo analítico epi- } \\
\text { demiológico. }\end{array}$ & Internação por TB. & $\begin{array}{l}\text { A.S. } \\
\text { A.E. } \\
\text { E. } \\
\text { V. }\end{array}$ & $\begin{array}{l}\text { Existe relação entre } \\
\text { risco para interna- } \\
\text { ção e baixa renda } \\
\text { familiar. }\end{array}$ & $\begin{array}{l}\text { Questões sociais e ambien- } \\
\text { tais devem ser amplamente } \\
\text { consideradas, pois, estão } \\
\text { relacionados às internações. }\end{array}$ \\
\hline
\end{tabular}




\begin{tabular}{|c|c|c|c|c|c|}
\hline $\begin{array}{l}\text { NEVES et al. } \\
(2012) \\
\text { Ribeirão Preto - SP }\end{array}$ & $\begin{array}{l}\text { Qualitativa com } \\
\text { realização de entre- } \\
\text { vistas }\end{array}$ & Morbidade. & $\begin{array}{l}\text { A.S. } \\
\text { A.E. } \\
\text { A.C. } \\
\text { Q.V. } \\
\text { E. } \\
\text { V. }\end{array}$ & $\begin{array}{l}\text { Baixa escolarida- } \\
\text { de é situação de } \\
\text { vulnerabilidade. A } \\
\text { qualidade de vida } \\
\text { e baixas condições } \\
\text { socioeconômicas es- } \\
\text { tão relacionadas aos } \\
\text { indicadores. }\end{array}$ & $\begin{array}{l}\text { Deve haver estratégias con- } \\
\text { juntas de várias políticas } \\
\text { para reduzir o impacto epi- } \\
\text { demiológico da doença. }\end{array}$ \\
\hline $\begin{array}{l}\text { BASTA et al. (2013) } \\
\text { Campo Grande - } \\
\text { MS }\end{array}$ & $\begin{array}{l}\text { Analise descritiva } \\
\text { epidemiológica }\end{array}$ & $\begin{array}{l}\text { Casos novos notifi- } \\
\text { cados }\end{array}$ & $\begin{array}{l}\text { A.S. } \\
\text { A.C. }\end{array}$ & $\begin{array}{l}\text { Houve desigualda- } \\
\text { des nas caracterís- } \\
\text { ticas relacionadas } \\
\text { ao adoecimento se- } \\
\text { gundo a raça/cor. A } \\
\text { população indígena } \\
\text { apresentou maiores } \\
\text { taxas. }\end{array}$ & $\begin{array}{l}\text { Os autores afirmam que } \\
\text { deve existir interesse políti- } \\
\text { co para reduzir a pobreza e } \\
\text { as desigualdades, caso con- } \\
\text { trário à população de risco } \\
\text { continuará vulnerável. }\end{array}$ \\
\hline $\begin{array}{l}\text { ERAZO et al. } \\
(2014) \\
\text { Salvador - BA }\end{array}$ & Estudo ecológico. & $\begin{array}{l}\text { Coeficiente de inci- } \\
\text { dência. }\end{array}$ & $\begin{array}{l}\text { A.S. } \\
\text { A.E. } \\
\text { V. }\end{array}$ & $\begin{array}{l}\text { Foram encontrados } \\
\text { mais casos em po- } \\
\text { pulações conside- } \\
\text { radas vulneráveis e } \\
\text { em regiões que con- } \\
\text { centram os bolsões } \\
\text { de pobreza. }\end{array}$ & $\begin{array}{l}\text { Medidas eficazes para o } \\
\text { controle da doença devem } \\
\text { ser direcionadas a melhorar } \\
\text { as condições de vida da } \\
\text { população. }\end{array}$ \\
\hline
\end{tabular}

*Legenda: A.C. - aspectos culturais, A.S. - aspectos sociais, A.E. - aspectos econômicos, E. - educação, Q.V. - qualidade de vida e V. vulnerabilidades.

Em estudo ecológico realizado por Hino et al. (2011b) foram identificados os casos novos de TB em Ribeirão Preto (SP) e constatou-se que os casos não eram uniformemente distribuídos nos bairros do município. As áreas que concentravam os maiores índices de TB foram aquelas que possuíam concentração de pessoas em situação de pobreza e algumas áreas com condições intermediárias de vida.

Dentre as publicações selecionadas para este estudo, aqueles envolvendo o método ecológico, associaram os casos de TB a regiões territoriais específicas. Foi possível notar que as áreas que apresentavam altos indicadores da TB eram aquelas com maiores níveis de desigualdade social, precárias condições de vida e moradia, altos níveis de pobreza, baixa renda, menor IDH e níveis de escolaridade ruins (GUIMARÃES. et al., 2012; HINO et al., 2011b; de QUEIROGA et al., 2012; RODRIGUES-JÚNIOR; RUFFINO-NETTO; CASTILHO, 2014; ROZA; CACCIA-BAVA; MARTINEZ, 2012; VENDRAMINI et al., 2010).

No entanto, alguns estudos apontam altos indicadores da doença em regiões com alto IDH localizadas em locais onde as condições de vida eram consideradas intermediárias a alta. Várias hipóteses foram levantadas pelos autores para justificar estes achados: subnotificações de casos, presença de bolsões de pobreza dentro dessas áreas, inexistência de equipamentos e/ou acesso aos serviços de saúde e o acometimento pela TB nas diversas classes sociais, considerando que a dinâmica social envolvida nas relações humanas diárias é suficiente para manter a cadeia de transmissão nas coletividades (ACOSTA; BASSANESI, 2014; ERAZO et al., 2014; De QUEIROGA et al., 2012; RODRIGUES-JÚNIOR; RUFFINO-NETTO; CASTILHO, 2014).

Desse modo, a doença deve ser compreendida como algo que se desenvolve em grupos sociais desfavorecidos e específicos capazes de causar prejuízos a toda sociedade. Portanto, será impossível avançar no controle da TB se não houver ações conjuntas entre os diversos setores do governo, em esforço combinado para reduzir as desigualdades, a pobreza e a exclusão social e para melhorar os equipamentos de saúde.

Historicamente o processo de mudança social e econômico no Brasil é lento. Considerando a distribuição do IDH no país nos últimos 60 a 70 anos notam-se poucas mudanças (RODRIGUES-JÚNIOR; RUFFINO-NETTO; CASTILHO, 2014).

Contudo, as transformações resultantes dos longos períodos de mudança social e da economia refletem em alterações na tendência da TB desde o século passado no Brasil. Os métodos de controle da doença, segundo Ruffino- Netto e Pereira (1981), podem ser classificados como específicos (vacinação, quimioprofilaxia, diagnóstico e tratamento) e inespecíficos (melhoria das condições de vida, alimentação, habitação, acesso a serviços de saúde) e ambos possuem considerável importância na tendência da TB ao longo da história. Os autores concordam que os efeitos dos fatores inespecíficos sobre a saúde da população, quando esta resulta em melhoria das condições de vida, reduzem o risco de morbimortalidade da TB e de várias outras doenças.

Apesar dos consideráveis recursos dispensados pelo governo federal em políticas sociais de ampliação do acesso a moradia, renda, alimentação e combate a fome e a miséria, são altos os indicadores de TB nas pessoas atendidas por esses programas caracterizando que estes, não atingiram o impacto esperado nas condições de vida dessas populações (DE OLIVEIRA; GONÇALVES, 2013).

\section{Relação entre indicadores epidemiológicos da tuberculo- se e aspectos culturais/comportamentais}

Aspectos culturais e comportamentais como os encontrados na população indígena de Mato Grosso (MT) e Mato Grosso do Sul (MS) e relacionados ao preconceito e estigma da doença da população brasileira, ainda são identi- 
ficadas em muitas publicações.

Em um estudo epidemiológico de mortalidade por TB realizado no MS ficou evidenciado que as maiores taxas de óbito estavam relacionadas às populações indígenas do estado. Essa população possui níveis alarmantes da doença no país e para mudar este cenário não bastam apenas intervenções específicas como cobertura vacinal, diagnóstico precoce e tratamento supervisionado. São necessárias ações políticas concretas de redução da pobreza, miséria e redução das desigualdades sociais das quais estas populações estão expostas (BASTA et al., 2013; LARROQUE et al., 2013).

Relatos de pacientes tuberculosos referindo isolamento social, angústia, preconceito e vergonha demonstram a fragilidade e negligência com que a doença é tratada no país, além da desinformação e falta de vínculo e apoio das equipes de atenção básica em ações de educação em saúde e educação permanente (HINO et al., 2011a; LEMOS et al., 2012; WELCH; COIMBRA, 2011).

De acordo com Welch e Coimbra (2011) apesar das diversidades interculturais existentes na população indígena, essas não foram apontadas como impeditivo para o desenvolvimento das ações de controle previstas pelo Programa Nacional de Controle da Tuberculose (PNCT). Os autores apontam que a população aceita ser vacinada, examinada e tratada conforme o modelo biomédico, apesar de culturalmente também associarem a doença a rituais de feitiçaria. Segundo eles a alta incidência da doença nessas populações está relacionada à falta de recursos, estrutura e acesso aos equipamentos de saúde em nível local, o que não significa necessariamente cobertura de equipes de saúde.

A cobertura de Equipes de Saúde da Família (ESF) infelizmente não garante indicadores melhores para TB, pois a baixa capacidade de resolução e a falta de recursos físicos e humanos comprometem a atenção oferecida. Todavia, quando as ESF utilizam o tratamento supervisionado como estratégia os indicadores tendem a melhorar consideravelmente (YAMAMURA et al., 2014).

Por questões culturais e sociais de estigma e preconceito alguns pacientes escondem a doença de familiares e amigos. Os profissionais de saúde devem compreender que apesar da doença ser encarada como rotina diária de seu trabalho, não é percebida da mesma maneira pelos doentes e familiares. Ela envolve mudanças no modo de viver e por este motivo é fundamental que haja escuta, discussões e trocas de experiências objetivando maior divulgação e informação a cerca do assunto (DE PAULA et al., 2014; SOUZA; DA SILVA; MEIRELLES, 2010).

Os sentimentos de angústia, medo e preconceito citados em vários estudos podem ser minimizados com maior ênfase ao vínculo e apoio. Segundo Hino et al. (2011a) a estratégia do Tratamento Supervisionado (TS) não visa apenas garantir a ingestão diária da medicação, mas acompanhar e apoiar o processo de tratamento, esclarecer dúvidas e participar ativamente do processo de cura dos pacientes.

Para tornar isso possível os profissionais devem contar com algumas ferramentas conhecidas por meio da política nacional de humanização, tais como: escuta qualificada, valorização das queixas, projetos terapêutico singular e coletivo, co-responsabilização e autonomia. Essas ferramentas tornam possível o conhecimento das reais necessidades e vulnerabilidades da população e convergem aos preceitos da promoção da saúde, proporcionando maior conhecimento e capacidade de enfrentamento e finalmente gerando a autonomia e o empoderamento almejado (BRASIL, 2006; BRASIL, 2009).

\section{Relação entre indicadores epidemiológicos da tuberculo- se e qualidade de vida}

A qualidade de vida foi outro aspecto utilizado para verificar o impacto da tuberculose na população, pois ela é resultante de uma complexa combinação de fatores biopsicossociais. Questões econômicas, culturais e sociais foram identificadas com os principais causadores de prejuízos à qualidade de vida. O medo associado ao preconceito e a preocupação excessiva com a saúde podem desencadear ansiedade desnecessária e comprometer o processo de adesão ao tratamento e cura (LEMOS et al., 2012).

O início do tratamento alivia os sintomas da doença e foi referido como importante componente relacionado à melhora da qualidade de vida dos pacientes, porém, apenas se considerarmos os aspectos físicos. Não houve melhora na qualidade de vida relacionada às questões psicológicas mesmo após o tratamento da doença aos pacientes coinfectados por TB/HIV, pois persistem sentimentos de exclusão, rejeição, culpa e desvalorização. A espiritualidade e/ou religiosidade foi associada com a manutenção e melhora na qualidade de vida dos pacientes com TB. Muitos pacientes buscam na espiritualidade a aceitação e conforto relacionados às suas dificuldades sociais, econômicas e de saúde (NEVES et al., 2012).

\section{Relação entre indicadores epidemiológicos da tuberculo- se e aspectos educacionais}

Em relação aos aspectos educacionais foi observada a relação entre aumento das chances de vulnerabilidade pela doença e baixa escolaridade na maioria dos estudos. A falta de informação e desconhecimento da doença associada à baixa resolutividade dos serviços de saúde tornam essas populações vulneráveis a TB, pois, dificultam o processo de procura por atendimento, aumentam as chances de propagação e manutenção da cadeia de transmissão da doença e criam maior estado de debilidade física aos doentes (ACOSTA; BASSANESI, 2014; De PAULA et al., 2014; HINO et al., 2011a; de QUEIROGA et al., 2012; NEVES et al., 2012; OLIVEIRA; GONÇALVES, 2013; ROZA; CACCIA-BAVA; MARTINEZ, 2012; VENDRAMINI et al., 2010).

A falta de percepção sobre a vulnerabilidade e situação de risco implica em redução do autocuidado. Os níveis de escolaridade baixo são associados ao aumento deste risco, no entanto, Vendramini et al. (2010) chama a atenção ainda, para o elevado número de analfabetismo no país e considera também o analfabetismo funcional um importante aspecto educacional relacionado à doença.

A educação pode ser considerada ponto fundamental para desencadear os demais fatores associados à vulnerabilidade, aos aspectos socioeconômicos, culturais e de qualidade de vida.

Por meio da educação é possível transformar uma situação de prejuízo da qualidade de vida, pois, ela é capaz de oferecer maiores chances de desenvolvimento econômico 
e organização social (o que contribui para manutenção de estado nutricional elevado, melhores condições vida, moradia e acesso a serviços básicos de saúde e saneamento), auxilia a compreensão do processo de saúde-doença (e a identificação dos sintomas e procura precoce por atenção), reduz as taxas de abandono do tratamento e aumenta as taxas de cura.

\section{Conclusão}

As publicações encontradas e selecionadas pelo presente estudo revelam que vários autores têm associado às pesquisas sobre a tuberculose às condições de vida das populações. A preocupação com as questões da promoção da saúde, tais como: alimentação/nutrição, emprego/renda, habitação, educação, economia e ambiente físico e social são discutidas e evidenciam o caráter fortemente social da doença.

Evidentemente que os indicadores da TB sofreram considerável alteração e queda ao longo da história do país, principalmente devido às transformações econômicas, sociais e culturais. Somado a isto, houve a criação de um sistema universal de saúde que conta ainda hoje com programas, ações e metas específicas para o controle, bem como o crescimento e fortalecimento dos grupos de pesquisa, garantindo melhor análise do panorama da doença.

Contudo, apesar do fortalecimento dos programas de controle da tuberculose, do aumento da cobertura de equipes de atenção básica e do maior número de pesquisas e estratégias voltadas a TB, os indicadores mostram que nas últimas décadas o Brasil vive um momento de estagnação, ou seja, não consegue reduzir seus indicadores.

Ainda de acordo com as publicações encontradas e em vista do atual cenário, os problemas relacionados à dificuldade de controle são muitos, desde aqueles envolvendo ações específicas, como a vacinação, quimioprofilaxia, busca ativa de sintomáticos respiratórios, diagnóstico precoce e tratamento, até aqueles que envolvem ações inespecíficas dirigidas à redução da pobreza extrema, desigualdade/exclusão social, precárias condições de moradia e baixos níveis de escolaridade.

Considerando que a promoção da saúde objetiva que os indivíduos possam ter maior conhecimento e domínio sobre os seus determinantes sociais e que, o Brasil possui uma enorme diversidade econômica, cultura e social, a educação de qualidade deve ser tratada como ponto de partida pois, somente deste modo é possível reverter grande parte das fragilidades e vulnerabilidade das quais a população está exposta.

Por fim, as mudanças almejadas dependem de ações concretas e dirigidas à redução da pobreza, investimentos em educação e garantia de oferta de serviços de saúde em quantidade suficiente e de qualidade, para reduzir não apenas os indicadores da TB como de várias outras doenças.

\section{Referências}

ACOSTA, L. M. W.; BASSANESI, S. L. O paradoxo de Porto Alegre: os determinantes sociais e a incidência da tuberculose. Rev Bras Epidemiol, v. suppl d.s.s., p. 88 $-101,2014$.
BASTA, P. C.; MARQUES, M.; OLIVEIRA, R. L. CUNHA, E. A. T.; RESENDES, A. P. C. SOUZASANTOS, R. Desigualdades sociais e tuberculose: análise segundo raçã/cor, Mato Grosso do Sul. Rev Saúde Pública, São Paulo, v. 47, n. 5, p. 854- 864, 2013.

BUSS P. A vigilância da saúde para a promoção da saúde. In: Czeresnia D, Freitas CM, organizadores. Promoção da Saúde - Conceitos, Reflexões, Tendências, Rio de Janeiro: Fiocruz; 2003. p. 148-158, 2000.

BRASIL. Ministério da Saúde. Fundação Nacional de Saúde. Tuberculose: guia de vigilância epidemiológica. Brasília, (DF): Ministério da Saúde, 2002.

BRASIL. Ministério da Saúde. Política Nacional de Promoção da Saúde 2006. Brasília, DF, Ministério da Saúde; 2006.

BRASIL. Ministério da Saúde. Secretaria de Vigilância em Saúde. Secretária de Atenção à Saúde. Política nacional de promoção da saúde Brasília, DF, 2007.

BRASIL. Ministério da Saúde. Secretaria de atenção a saúde. Política Nacional de Humanização da Atenção e Gestão do SUS. Brasília, DF, Ministério da Saúde, 2009.

BRASIL. Ministério da Saúde. Secretaria de Vigilância em Saúde. Programa Nacional de Controle da Tuberculose. Brasília,(DF): Ministério da Saúde, 2010.

De QUEIROGA, R. P. F.; de SÁ. L. D.; NOGUEIRA, J. A.; de LIMA, E. R. V.; SILVA, A. C. O.; PINHEIRO, P. G. O. D.; BRAGA, J. U. Distribuição espacial da tuberculose e a relação com condições de vida na área urbana do município de Campina Grande- 2004 a 2007. Rev Bras Epidemiol, v. 15, n. 1, p. 222- 232, 2012.

De OLIVEIRA, N. F.; GONÇALVES, M. J. F. Fatores sociais e ambientais associados à hospitalização de pacientes com tuberculose. Rev Latino-Am Enfermagem, v. 21, n. 2 , p. $1-8$, mar-abr, 2013.

De PAULA, R.; LEFEVRE, F. LEFEVRE, A. M. C.; GALESI, V. M. N.; SCHOEPS, D. Por que os pacientes de tuberculose procuram as unidades de urgência e emergência para serem diagnosticadas: um estudo de representação social. Rev Bras Epidemiol, p. 600-614, jul-set, 2014.

De SOUZA, S. De S.; Da SILVA, D. M. G. V.; MEIRELLES, B. H. S. Representações sociais sobre a tuberculose. Acta Paul Enferm, São Paulo, SP, v. 23, n. 1, p. 23-28, 2010.

ERAZO, C.; PEREIRA, S. M.; COSTA, M. daC. N.; EVANGELISTA-FILHO, D. BRAGA, J. U.; BARRETO, M. L. Tuberculosis and living conditions in Salvador, Brazil: a spatial analysis. Rev Panam Salud Publica, v. 36, n. 1, 2014.

GUIMARÃES, R. M.; LOBO, A. de P.; SIQUEIRA, E. A.; 
BORGES, T. F. F.; MELO, S. C. C. Tuberculose, HIV, e pobreza: tendência temporal no Brasil, Américas e mundo. J Bras Pneumol, Brasília, DF, v. 38, n. 4, p. 511-517, 2012.

HINO, P.;TAKAHASHI, R. F.; BERTOLOZZI, M. R.; EGRY, E. Y. As necessidades de saúde e vulnerabilidades de pessoas com tuberculose segundo as dimensões acesso, vínculo e adesão. Rev Esc Enferm USP, São Paulo, SP, v. 45, n. esp. 2, p. 1656-1660, 2011a.

HINO, P.; VILLA, T. C. S.; da CUNHA, T. N.; dos SANTOS, C. B. Padrões espaciais da tuberculose e sua associação à condição de vida no município de Ribeirão Preto. Ciência e Saúde Coletiva, São Paulo, SP, v. 16, n. 12, p. 4795- 4802, 2011 b.

KRITSKI, A.; CONDE, M, B.; SOUZA, G. R. M.; Tuberculose: do ambulatório à enfermaria. 3. ed. São Paulo: Atheneu, 2005.

LARROQUE, M. M.; PONTES, E. R. J. C.; MARQUES, A. P. Da C.; FERNANDES, S. M. Mortalidade por tuberculose: municípios prioritários de Mato Grosso do Sul, 1999- 2008. Arq Ciênc Saúde UNIPAR, Umuarama, v. 17, n. 3, p. 163-169, set-dez, 2013.

LEMOS, L. De A.; FEIJÃO, A. R.; GIR, E.; GALVÃO, M. T. G. Aspectos da qualidade de vida de pacientes com coinfecção HIV/tuberculose. Acta Paul Enferm, São Paulo, SP, v. 25, n. Especial, p. 41-47, 2012.

MOREIRA, C. M. M.; ZANDONABE, E.; DIETZE, R.; MACIEL, E. L. N. Tuberculosis related mortality in the state of Espírito Santo, Brazil, 1985-2004. J Bras Pneumol, Brasília, DF, v. 34, n. 8, p. 601-606, 2008.

NEVES, L.A. de S.; CANINI, S. R. M.; REIS, R. K.; dos SANTOS, C. B.; GIR, E. Aids e tuberculose: a coinfecção vista pela perspectiva da qualidade de vida dos indivíduos. Rev Esc Enferm USP, São Paulo, SP, v. 46, n. 3, p. 704$710,2012$.

PIVA, S. G. N.; COSTA, M. da C. N.; BARRETO, F. R.; PEREIRA, S. M. Prevalência de deficiência nutricional em pacientes com tuberculose pulmonar. J Bras Pneumol, Brasília, DF, v. 39, n. 4, p. 476- 483, 2013.

RODRIGUES-JUNIOR, A.L.; RUFFINO-NETTO, A.; de CASTILHO, E. A. Distribuição espacial do índice de desenvolvimento humano, da infecção pelo HIV e da comorbidade AIDS- tuberculose: Brasil, 1982-2007. Rev Bras Epidemiol, v. suppl d.s.s., p. 204- 215, 2014.

ROZA, D. L.; CACCIA-BAVA, M. do C. G. G.; MARTINEZ, E. Z. Spatio-temporal patterns of tuberculosis incidence in Ribeirão Preto, State of São Paulo, southeast Brazil, and their relationship with social vulnerability: a Bayesian analysis. Rev Soc Bras Med Trop, Ribeirão Preto, SP, v. 45, n. 5, p 607-615, sep-oct, 2012. tuberculose e condições de vida: o caso Rio de Janeiro. Rev Saúde em Debate, Rio de Janeiro, RJ, n. 12, p. 27-34, 1981.

SANTOS, J. Resposta brasileira ao controle da tuberculose. Rev Saúde Pública, São Paulo, v. 41, supl. 1, p. 89-94, 2007.

VENDRAMINI, S. H. F.; GAZETA, C. E.; CHIARAVALOTTI NETTO, F.; CURY, M. R.; MEIRELLES, E. B.; KUYUMJIAN, F. G.; VILLA, T. C. $\mathrm{S}$. Tuberculose em município de porte médio do sudeste do Brasil: indicadores de morbidade e mortalidade, de 1985 a 2003. J Bras Pneumol, Brasília, DF, v. 31, n. 3, p. 237-243, maio. jun. 2005.

VENDRAMINI, S. H. F.; dos SANTOS, N.S.G.M.; SANTOS, M. de L. S. G.; CHIARAVALLOTI-NETO, F.; PONCE, M. A. Z.; GAZETTA, C. E.; VILLA, T. C. S. V.; RUFFINO-NETTO, A. Análise espacial da co-infecção tuberculose/HIV: relação com níveis socioeconômicos em município do sudeste do Brasil. Rev Soc Bras Med Trop, Ribeirão Preto, SP, v. 43, n. 5, p. 536-541, set-out, 2010.

WELCH, J. R.; COIMBRA-JUNIOR, C. E. A. Perspectivas culturais sobre transmissão da tuberculose entre os Xavánte de Mato Grosso, Brasil. Cad Saúde Pública, Rio de Janeiro, RJ, v. 27, n. 1, p. 190-194, jan, 2011.

WORLD HEALTH ORGANIZATION. The Global Plan to Stop TB 2006-2015: actions for life toward a world free of tuberculosis. Geneva ; 2006.

YAMAMURA, M.; NETO, M. S.; FREITAS, I. M.; RODRIGUES, L. B. B.; POPOLIN, M. P.; UCHOA, S. A. C.; FRONTEIRA, I.; ARCÊNCIO, R. A. Tuberculose e iniquidade social em saúde: uma análise ecológica utilizando técnicas estatísticas multivariadas, São Paulo, Brasil. Rev Panam Salud Publica, v. 35, n. 4, 2014 